\title{
La investigación sobre educación como desplazamiento: no saber, abrirse, devenir
}

\section{Research on education as displacement: not knowing, opening up, becoming}

\author{
Aurelio Castro Varela ${ }^{1}$ \\ aurelio.castro@ub.edu \\ Judit Onsès Segarra \\ jonsesse@gmail.com \\ Universidad de Barcelona, España
}

\section{Resumen:}

El paraguas poscualitativo conlleva diferentes desplazamientos en el modo de comprender la investigación. Algunas nociones -como diseño del proyecto, recogida o análisis de datos, entrevistas, observación, representación, etc.- se ponen en cuestión al tiempo que aparecen otras nuevas como alternativa. ¿Pero qué efectos tiene esta reconceptualización durante el trabajo de campo? Este artículo establece un diálogo entre dos tesis doctorales para dar cuenta de ese giro. La primera de ellas etnografía la actividad educativa de un cinefórum vinculado a la Asamblea de Vecinas de Poble Sec. La segunda cartografía los fenómenos de aprendizaje relacionados con la documentación visual y los procesos de trabajo de estudiantes de quinto de primaria en una escuela de Barcelona. En el primer caso la necesidad de "devenir investigador" -en vez de la idea antropológica clásica de "going native" - entrañó su principal desafío metodo-

\begin{abstract}
:
The post-qualitative umbrella implies several displacements regarding the way research is understood. Some notions such as project design, data gathering or analysis, interviews, observation, representation, etc. are questioned while some others appear as sound alternatives. But what effects does this reconceptualization have during fieldwork? The present article establishes a dialogue between two doctoral dissertations to answer this question. The first thesis traces an ethnography on the learning activity of a cinema forum linked to the Assembly of Residents of Poble Sec. The second maps learning phenomena connected with the visual documentation and the work processes by $5^{\text {th }}$ grade primary students in a school in Barcelona. In the former thesis the main methodological challenge was how to approach the process of becoming a researcher in contrast to the classical anthropological idea of "going native"; a contorted research figure
\end{abstract}

1 Dirección para correspondencia (correspondence address):

Aurelio Castro Varela. Universidad de Barcelona. Departamento de Artes Visuales y Diseño.. Facultad de Bellas Artes. C/ Adolf Florensa, 8. 08028 Barcelona (España). 
lógico; una figura investigadora en torsión sostuvo, desde la precariedad y la falta de tiempo, ese tránsito. En el segundo el desplazamiento consistió en abrirse a otras dimensiones como la improvisación, los movimientos, las intra-acciones (Barad, 1996, 2003, 2007) y las líneas de fuga (Deleuze y Guattari, 2004) de la propia indagación. En ambas investigaciones se puso en juego ese estado de incerteza y apertura como condición y forma de conocimiento acerca de espacios pedagógicos. ¿Cómo la posición del investigador en educación se presenta como un lugar de "no saber" (Rogoff, 2006; Atkinson, 2011, 2012, 2017), de descentramiento epistemológico y de devenir subjetivo en las situaciones y casos de estudio (Barad, 2003, 2007)?

\section{Palabras clave:}

Investigación poscualitativa; devenir investigador; desplazamiento; no saber; etnografía; cartografía; investigación sobre educación. sustained this transit so as to deal with precariousness and lack of time. In the latter thesis displacement consisted of opening up the research that was being undertaken to other dimensions such as improvisation, movements, intra-actions (Barad, 1996, 2003, 2007) and its own lines of flight (Deleuze and Guattari, 2004). Both theses displayed a state of uncertainty and opening that emerged as the condition and the form of knowledge about pedagogical spaces. How do the positions of researchers in education become places of "not knowing" (Rogoff, 2006; Atkinson, 2011, 2012, 2017), places of epistemic decentering and subjective becoming within specific situations and case studies (Barad, 2003, 2007)?

\section{Keywords:}

Post-qualitative research; researcher becoming; displacement; not knowing; ethnography; cartography; learning research.

\section{Résumé:}

Le parapluie post-qualitatif implique plusieurs changements de la manière de comprendre la recherche. Quelques notions telles que le plan du projet, la collecte ou l'analyse des données, des entrevues, l'observation, la représentation, etc., sont mises en question tandis que de nouvelles notions apparaissent comme alternatives. Mais quels effets cette reconceptualisation a-t-elle sur le travail de champ ? Cet article propose le dialogue entre deux thèses de doctorat pour en rendre compte. La première fait une ethnographie de I'activité éducative d'un cineforum lié à l'Assemblée de voisines de Poble Sec. La seconde cartographie les phénomènes d'apprentissage liés à la documentation visuelle et les processus de travail des élèves de cinquième année dans une école à Barcelone. Dans le premier cas, la nécessité de " devenir chercheur »-au lieu de l'idée anthropologique classique de " going native »-a entraîné son principal défi méthodologique ; une figure $\mathrm{d}^{\prime}$ investigateur en torsion a soutenu ce transit sous la précarité et le manque de temps. Dans le second, le déplacement consistait à s'ouvrir à d'autres dimensions telles que l'improvisation, le mouvement, intra-actions (Barad, 1996, 2003, 2006), et les lignes de fuite (Deleuze et Guattari, 2004) de l'enquête elle-même. Dans les deux investigations l'incertitude et l'ouverture comme condition et forme de connaissances sur les espaces d'apprentissage sont entrées en jeu. Comment la position du chercheur dans l'éducation se présente-t-elle comme un lieu de " ne pas savoir »(Rogoff, 2006; Atkinson, 2011, 2012, 2017), de décentrage épistémologique et de devenir subjectif dans les situations et études de cas (Barad, 2003, 2007)?

\section{Mots clés:}

Recherche post-qualitative; devenir investigateur; déplacement; ne pas savoir; ethnographie; cartographie; recherche sur éducation.

Fecha de recepción: 22-11-2018

Fecha de aceptación: 15-3-2019 


\section{Introducción}

De orientación poscualitativa, los nuevos materialismos y empirismos se presentan como un proyecto ético-onto-epistemológico y metodológico (St. Pierre, Jackson y Mazzei, 2016). Este adjetivo compuesto hace referencia a un nuevo modo de investigación que reconecta las formas de conocimiento, el ser de aquello sobre lo que se indaga y, al mismo tiempo, la manera en que consideramos el anudamiento práctico de ambos: "We focus on two of those conditions we think are especially important: an ethical imperative to rethink the nature of being [...] and a heightened curiosity and accompanying experimentation" (ibid., p. 100; énfasis en el original).

En consecuencia, dicho replanteamiento pasa en primer lugar por establecer una comprensión ontológica cuyas premisas se repiten y acumulan en multitud de trabajos y presentaciones académicas. Los a priori modernos que jerarquizan lo humano sobre lo no humano, lo discursivo sobre lo material, o la cultura sobre la naturaleza, y que fijan además una oposición binaria entre cada uno de ellos, se quiebran (ver Barad, 2003; Lather y St. Pierre, 2013; St. Pierre, Jackson y Mazzei, 2016; St. Pierre, 2016; entre un largo etcétera). Este desplazamiento, a su vez, presenta implicaciones éticas:

If humans have no separate existence, if we are completely entangled with the world, if we are no longer masters of the universe, then we are completely responsible to and for the world and all our relations of becoming with it. We cannot ignore matter (e.g., our planet) as if it is inert, passive, and dead. It is completely alive, becoming with us, whether we destroy or protect it (St. Pierre, Jackson and Mazzei, 2016, p. 101).

Sin embargo, el paso epistémico y ético hacia lo que se conoce como una "ontología del devenir", fundamentada principalmente en la filosofía de Gilles Deleuze o Karen Barad y según la cual la realidad sería entendida

"as a continual process of flux or differentiation even though this fact is usually masked by powerful and pervasive illusory discourses of fixity, stability, and identity that have characterized most of western philosophy and theory since at least the Enlightenment" (Martin y Kamberelis, 2013: 670), 
no parece tan sencillo. Si el post de la investigación poscualitativa sugiere movimiento, trayectorias y fluidez, cabe preguntarse -señala Jennifer C. Greene (2013)- "from where and, perhaps more importantly, to where and to what" (p. 759):

Without a shared understanding of how some knowledge or insight or understanding about the world is obtained - shared within the varied communities of scholars, and more importantly between researchers and our audiences how will such knowledge or understanding be warranted? And how will it be of any meaningful or constructive consequence in the world? I worry about a loss of the systematic character of social inquiry, where the systems (guidelines, rules, precepts, assumptions) are open and available to all. I worry that inquiry then becomes less distinguishable from other important human endeavors and less uniquely consequential. (p. 753)

Asimismo, Greene se pregunta "about the privileging of philosophy over practice" en los textos sobre investigación poscualitativa (ibid., p. 755). Este artículo trata justamente de efectuar, a partir del diálogo entre dos trabajos doctorales que han debido mantenerse en un desplazamiento constante, ese paso hacia la práctica. De esta manera pretendemos llegar a comprender cómo el proyecto onto-ético-epistemológico y metodológico que dicen abrir los nuevos materialismos se concreta, más allá de una continua declaración axiomática, en problemáticas y situaciones compartibles y significativas. En el primer caso, se corresponde con la emergencia de la investigación y su correlación con una posición "nativa" en el campo, la cual condujo a una indagación descriptiva sobre el modo de existencia y las formas asociativas que caracterizaban al caso de estudio: el Cinefórum de la Asamblea de Vecinas de Poble Sec, en Barcelona. En el segundo caso, experimentar con las formas de investigar y con la documentación visual sobre procesos de aprendizaje en una escuela llevó a pensar de manera diferente ese conjunto de relaciones que sostienen tanto la producción de conocimiento como las prácticas educativas.

\section{Caso 1. Devenir investigador (Aurelio Castro)}

Frente al movimiento clásico -epistémico y metodológico- en que se ha basado la etnografía, conocido como "going native" y consistente en 
una participación empática por parte del etnógrafo a fin de captar mejor el punto de vista del grupo social investigado ${ }^{2}$, el desplazamiento que funda este trabajo doctoral parece haber sido precisamente el contrario. Devenir investigador, en vez de nativo, constituyó el pulso de la investigación en las dos acepciones que tiene el término: enfrentamiento de posiciones y, al mismo tiempo, lo que la hacía latir como tal.

En junio de 2011, el Movimiento 15M decidía poner fin a la ocupación masiva de Plaza Catalunya y diseminarse por el resto de Barcelona ${ }^{3}$. En el barrio de Poble Sec, muy próximo al centro de la ciudad y parte del distrito Sants-Montjuïc, se estableció poco después una asamblea popular dotada de varias comisiones de trabajo (economía, comunicación, trabajo en la calle, etc.) y resuelta a introducir en la vida cotidiana de dicho territorio protestas y contrainformación a propósito de la crisis que estábamos padeciendo. A este efecto, a principios de 2012, organizó también un cinefórum para discutir colectivamente cuestiones de actualidad política o repensar su papel en el tejido urbano. El pase mensual de una película en varios lugares públicos -como el Centro Cívico del Sortidor o la Casa del Mar- daba pie a un debate abierto a cualquiera y dispuesto en un círculo de tipo asambleario, durante el cual las imágenes y sonidos proyectados se situaban en un contexto local a través de un cierto ejercicio de "traducción" ${ }^{4}$.

2 "The phrase 'going native' as a researcher is often attributed to Bronislaw Malinowski in his reflections on the relationship between the anthropologist and the objects of study in ethnographic fieldwork (Malinowski, 1922). On the basis of his extensive experience living in New Guinea from 1914 to 1918 Malinowski suggested that 'to grasp the native's point of view, his relations to life, to realize his vision of his world' (p. 290) anthropologists should 'go native;' that is, scientists in a foreign milieu should emphasize their roles as "participants" rather than "observers" to enhance their study of native peoples and cultures" (Kahuna, 2000, p. 439).

3 "La tendencia es la disgregación del movimiento y su traslado a las asambleas de barrios y municipios. A partir de ahora, serán los barrios los que canalicen los esfuerzos y organicen las protestas como la acampada organizada para el miércoles, frente al Parlament de Catalunya para impedir la aprobación de los presupuestos catalanes de 2011, y la manifestación del 19 de junio". En http://www.publico.es/espana/381399/ la-mayoria- de-indignados-acuerdan-no-acampar-por-la-noche-en-plaza-catalunya.

4 Por ejemplo, el visionado de Veinte años no es nada (Joaquim Jordà, 2005) llevó a cuestionar el relato pacificador de la Transición española y la ocultación de toda una serie de luchas políticas que se dieron a finales de los años 70 y principios de los 80; mientras que Videogramas de una revolución (Andrej Ujica y Harun Farocki, 1992), sobre la caída del régimen de Nicolae Ceaucescu en Rumanía en 1989, sirvió para examinar la producción simbólica de violencia tras la huelga general del 29 de marzo de 2012. 
Así pues, el cinefórum se acabó convirtiendo en un espacio pedagógico que conectaba el mundo de la asamblea con la cotidianidad de Poble Sec, y que utilizaba el dispositivo fílmico para efectuar periódicamente esta aproximación. Tal y como señala Elizabeth Ellsworth (2005), el lugar en el que acontece un aprendizaje no se sitúa necesariamente en instituciones formales y puede resultar "peculiar, irregular, abnormal or difficult to classify" (p. 5). Además, en la medida en que proporciona entornos y experiencias que actúan como "pivot point between movement/ sensation and thought", esa localización también abre la posibilidad de un devenir subjetivo de las participantes "in the making" (p. 8). Ver una película, en este sentido, "has a material nature that involves biological and molecular events taking place in the body of the viewer and in the physical and imagined space between the viewer and the film" (p. 6). ¿Cómo investigar pues ese "acontecimiento" humano y material que, en el caso del cinefórum, trataba de producirse una o dos veces al mes en diferentes puntos del barrio?

Por mi parte, participé en la Asamblea de Vecinas de Poble Sec desde que se convocó por primera vez en la calle Blai, frente a la biblioteca municipal, en junio de 2011. También me vinculé a su cinefórum después de la primera sesión, celebrada el 9 de febrero de 2012 en la Casa del Mar ${ }^{5}$. Todo ello fue asimismo algunos meses antes de empezar a redactar el proyecto de tesis que habría de entregar a finales de ese curso, y sin haberme percatado tampoco de que tales sesiones de cine se podían convertir en uno de los casos de estudio de una investigación doctoral que se tituló, finalmente, Estética de la proyección audiovisual. Asamblea, ficción y derecho a la ciudad en Poble Sec, Barcelona. En definitiva, formaba parte del grupo de personas (y cosas) que iba a investigar antes de saber siquiera que lo eran, ni cómo trataría de hacerlo.

Esta posición "en torsión" -es decir, la resultante de coorganizar las sesiones del cinefórum a la vez que investigaba, poco después, su desarrollo relacional, material y pedagógico- repercutió sobre todo en mi disponibilidad para elaborar un diario de campo durante el transcurso de las sesiones ${ }^{6}$. Sin embargo, esta dificultad metodológica entrañaba una

5 Se trata de un inmueble público, situado en los confines de Poble Sec con la zona portuaria de Barcelona, que depende del Departament de Benestar Social i Familia de la Generalitat de Catalunya. Su auditorio, donde tuvo lugar la proyección, suele hospedar congresos y actos institucionales.

6 El Cinefórum de la Asamblea de Poble Sec montó en total seis proyecciones: Interferències (Pablo A. Zareceansky, 2011) en la Casa del Mar (09.02.2012); Veinte años 
más amplia, de orden epistemológico. Aunque mi vinculación "nativa" a las actividades del grupo me proporcionaba un acceso sin restricciones a su práctica, el desarrollo de una agenda investigadora suplementaria a ese proceso tenía que lidiar con la falta de tiempo y, sobre todo, con no saber qué distancia -o quizá, qué otro tipo de proximidad- debía tomar con el caso de estudio. En suma, ¿qué cuestiones podía y quería hacerme en una escena de campo cuyo punto de partida no era en ningún caso el que la antropología había asumido como convencional??

Finalmente, la imposibilidad de investigar desde un lugar distanciado, o acaso de devenir investigador soslayando mi función de coorganizador, me llevó a elaborar en cada sesión una mirada situada y fundamentalmente descriptiva del ensamblaje de elementos humanos y no humanos que requería cada sesión del cinefórum. Si en tanto que "nativo" me preocupaba -junto al resto del grupo motor- por la película que íbamos a proyectar, a quien invitaríamos al debate, la disponibilidad de un determinado edificio para alojar la sesión, o los problemas técnicos que implicaba cada espacio; en tanto que investigador, no obstante, hacía seguimiento de los objetos, las prácticas y las conexiones que daban lugar a tales acciones y el modo de existencia que sostenía esa pedagogía fílmica.

Devenir investigador del cinefòrum implicaba, paradójicamente, el no poder serlo del todo o únicamente, y el tener que mantenerme en ese movimiento constante entre dos orillas que se traducía, del lado de la

no es nada en el Centro Cívico del Sortidor (23.02.2012); Pronto, espero (Chris Marker y Mario Marret, 1968) y Vuelta al trabajo en la fábrica Wonder (Jacques Willemont, 1968) de nuevo en el CC. Sortidor (27.03.2012); Videogramas de una revolución en el centro social Ateneu Rebel (10.05.2012); y una sesión al aire libre de cine mudo en la plaza de Santa Madrona (17.07.2012). A menudo debía tomar las notas de campo en casa, un día después de cada sesión y ya liberado de su sostenimiento. En las dos primeras, dado que aún no sabía que aquel era mi caso de estudio, las tuve que elaborar varias semanas después (aproximadamente en junio). A finales de año revisé y completé la información que figuraba en ellas con seis entrevistas en profundidad: la mitad a miembros del grupo motor de las proyecciones, y la otra mitad a espectadoras habituales. Además, por entonces ya había informado a las otras participantes del cinefórum de que una parte de mi investigación doctoral iba a tratar sobre nuestra experiencia compartida.

7 Con todo, la escena "clásica" del trabajo de campo etnográfico también ha sido recientemente cuestionada (vid. Holmes y Marcus, 2008; Marcus, 2013), sobre todo en el Estado español a raíz de diversas colaboraciones entre antropólogos y grupos activistas surgidas al calor del Movimiento 15M (vid. Corsín, 2014; Corsín y Estalella, 2013, 2016; Estalella y Sánchez Criado, 2016; Lafuente, Alonso, y Rodríguez, 2013). 
investigación, en un tipo de criticismo que "would try not to judge but to bring an oeuvre, a book, a sentence, an idea to life": "It would multiply not judgments but signs of existence" (Foucault, 1997, p. 323).

\section{Caso 2. Una investigación rizomática desde las teorías 'post' (Judit Onsès)}

Esta tesis es un corte de un segmento de mi vida. Este segmento contiene un tallo difuminado inicial, es decir, qué condicionantes y circunstancias previas a la tesis se estaban dando justo cuando empecé -oficialmente cuando me matriculé el primer curso de doctorado-, y un tallo difuminado final, que es cuando deposito esta tesis. Por tanto, esta tesis es un fragmento del rizoma que es mi vida y así será compartida, desde mi multiplicidad y desde el devenir. Eso significa que no es una tesis cerrada en sí misma, no es una tesis acabada y redonda, no es una tesis estática. Es una tesis que se está construyendo, antes y después de los cortes mencionados, y con sus movimientos, y velocidades, y líneas de fuga, y contradicciones, y limitaciones, y luces, y sombras, y llenos y vacíos, y..., y..., y... (Onsès, 2018, pp. 57-58).

En el caso de la investigación en la que se basa esta tesis doctoral, el paraguas poscualitativo (p. ej. Lather, 1990; Lather y St. Pierre, 2013; St. Pierre, 2011, 2013, 2014) conllevó diferentes desplazamientos en el modo de comprender y hacer investigación, entendiéndola desde el principio como un rizoma y desarrollándola rizomáticamente. Es decir, entendiendo el fenómeno investigativo y el fenómeno educativo como una maraña de sujetos, objetos, espacios, conceptos, tiempos y pensamientos que constantemente se afectan mutuamente, (re)configurando el propio fenómeno.

Según Deleuze y Guattari (2004, p. 25), un rizoma

conecta cualquier punto con otro punto cualquiera... No está hecho de unidades, sino de dimensiones, o más bien de direcciones cambiantes. No tiene ni principio ni fin, siempre tiene un medio por el que crece y desborda... el rizoma sólo está hecho de líneas: líneas de segmentaridad, de estratificación, como dimensiones, pero también línea de fuga o de desterritorialización como dimensión máxima según la cual, siguiéndola, la multiplicidad se metarmorfosea al cambiar de naturaleza... El rizoma es una antigenealogía... 
procede por variación, expansión, conquista, captura, inyección... el rizoma está relacionado con un mapa que debe ser producido, construido, siempre desmontable, conectable, alterable, modificable, con múltiples entradas y salidas, con sus líneas de fuga.

El inicio del doctorado y de la investigación en este caso se dio por una confluencia de factores: yo quería pedir una beca predoctoral. Para ello, necesitaba estar vinculada a un grupo de investigación y a un proyecto. Ese mismo año, el grupo de investigación consolidado Esbrina había empezado el proyecto europeo Do It Yourself in Education: Expanding Digital Competence To Foster Student Agency And Collaborative Learning (DIYLab; vid. www.diylab.eu). El objetivo principal del proyecto -que se desarrolló entre 2014 y 2016-fue

incorporar, en las instituciones implicadas, modalidades de aprender relacionadas con la cultura Do it Yourself (DIY) ... [es decir], introducir (en cada institución implicada) modos de acción y prácticas de enseñanza y aprendizaje surgidas en contextos informales [con el fin de] avivar el compromiso de los estudiantes proponiendo experiencias de aprendizaje colaborativas y significativas (Domingo-Coscollola, Onsès-Segarra y Sancho-Gil, 2018, p. 493, claudators míos, énfasis en original).

Yo venía de haber investigado en el Trabajo Final de Máster las posibilidades y potencialidades de la documentación visual en los procesos de aprendizaje en las clases de secundaria, a la vez que compartía parte de los intereses investigativos del grupo Esbrina. Tal vez podía seguir esa investigación vinculándome al proyecto DIYLab, trabajando con una de las escuelas de primaria participantes, y aportando esta dimensión de documentación visual relacionada con el aprendizaje de los jóvenes. De este modo, la investigación no partió de cero, sino que, por un lado, se conectó a ramificaciones que ya existían -un grupo de investigación existente y un proyecto existente- $y$, por otro, empezó a crear nuevas ramificaciones, bulbos y tubérculos: un contrato predoctoral con la Universidad de Barcelona, una investigación con estudiantes de primaria, y mi incorporación al grupo de investigación consolidado Esbrina.

Un rizoma no sólo conlleva no tener principio ni final, sino también descentramientos (desterritorializaciones). En este caso, las desterritorializaciones y reterritorializaciones fueron deviniendo y creando rizoma a 
lo largo de la investigación con la escuela de primaria. Ya desde un principio empezó con un planteamiento descentrado: en lugar de poner el foco en los estudiantes como 'objetos de investigación', se les in-corporó como investigadores y creadores de su propia realidad. Esto es, se les dio cámaras de video y fotografía para que documentaran visualmente sus procesos de trabajo y aprendizaje en el aula. A su vez, yo, como investigadora, también estaría documentando visualmente la vida en el aula.

Esto significó que durante la implementación del proyecto DIYLab, no sólo yo grabaría lo que allí sucediera, sino que los estudiantes de primaria , por grupos de tres a cinco, también documentarían visualmente sus procesos de aprendizaje. De este modo, se generó un descentramiento y multiplicidad en la mirada. Se huyó de una mirada centralizada, comúnmente extendida en los proyectos e investigaciones basados en la documentación visual (p. ej. Hoyuelos, 2007; Krechevski, Mardell, Rivard y Wilson, 2013; Lia, Iozzelli, Davoli y Tognetti, 2013; Xarxa Territorial d'Educació Infantil a Catalunya, 2009a, 2009b), donde es la docente o la investigadora solamente quien documenta visualmente el quehacer de los chicos y chicas, con el riesgo de monitorización y vigilancia que supone (Sánchez de Serdio, 2010), para proponer una mirada múltiple y relacional. La investigadora era entendida como un sujeto-en-relación, intra-actuando con el fenómeno de documentación visual y procesos de aprendizaje, junto con los estudiantes, la escuela, las maestras implicadas, los tiempos, espacios, materiales, discursos, imaginarios, etc.

$\mathrm{Y}$ estos modos de pensar, hacer y sentir se contagiaron en los modos de decir y escribir la tesis. Ésta también huyó de una autoría centralizada al desplazarse hacia una autoría relacional, pues lo escrito no era algo que 'salía de mí', sino que yo escribía poniendo en relación lo sucedido, lo activado, lo pensado, lo desconocido, etc. Escribía desde una posición enmarañada con los fenómenos de aprendizaje, una posición ontoepistemológica, metodológica y ética basada en la posición de Barad (1996, 2003, 2007) y la lógica del devenir de Deleuze y Guattari (2004). De acuerdo con Barad (1996, 2003),

la realidad no está compuesta de cosas o sujetos, sino de fenómenos, entendiendo los fenómenos como una maraña de espacios, tiempos y materias, que constantemente se están reconfigurando, enmarañando, relacionando y rearticulando según sus intra-acciones y las intra-acciones que se dan con otros fenómenos (Onsès, 2018, p. 14). 
Al comprender la realidad como múltiples fenómenos que están intraactuando, y entendiendo la intra-acción como esos modos de relación donde los espacios, los tiempos y lo matérico se afectan y (re)configuran mutuamente, se afectan entre ellos y afectan la realidad, la investigación se entendió desde la lógica del devenir, donde no hay una realidad fija, sino que la realidad está constantemente deviniendo. Se despliega en diferentes planos, no se rige ni por una jerarquía ni por una linealidad temporal. No hay lógica binaria y de opuestos, sino una lógica basada en sistemas abiertos (Capra, 2003; Tartás y Guridi, 2013), en hacer sobre la marcha (Rajchman, 2007; Taylor, 2016). Donde la investigación se convierte en algo vivo (Springgay, Irwin, y Wilson, 2005) y conectado a la vida (Braidotti, 2015).

Durante la implementación del proyecto DIYLab en las clases de primaria, la documentación visual en el aula pronto supuso un imposible. Los chicos y chicas trabajaban en pequeños grupos, se movían, se preguntaban entre ellos, hablaban. Con el material de grabación del que disponíamos, no podíamos hacer un buen registro del audio. Decidimos movernos, salir del aula, y organizar entrevistas por pequeños grupos de estudiantes en diferentes espacios de la escuela. Nuevo desplazamiento. Desterritorializar la investigación, los modos de crear material para la investigación, los modos de relación con la escuela, con los chicos y chicas, con las maestras. El despacho de profesores, la escalera de paso, la escalera de incendios, el aula de bachillerato, el aula de plástica... todos esos espacios deviniendo set de grabación. Estábamos resignificando los espacios educativos y de la escuela, desterritorializándolos. Habitarlos desde una posición de documentación visual, con las interrupciones, movimientos, afectos e irrupciones que se activaron. Adoptando una actitud de wonder y wander (Atkinson, 2018).

Wonder como ese estado mezcla de sorpresa, de estar abierto a la sorpresa, de dejarse sorprender pero desde una actitud interrogadora, abierta al pensar $y$ al pensamiento que se puede generar en el mismo pensar. $Y$ wander por ese... permitirse deambular, vagar por los planos de realidad (Onsès, 2018, p. 183, énfasis en original).

Y esta actitud estaba en el fenómeno investigativo y de escritura de tesis; los estudiantes, yo, los espacios, los tiempos, lo matérico (las cámaras, las grabaciones, las mesas, sillas, ventanas, discursos, pensamientos, 
inseguridades, etc.), todos estaban bañados -o enmarañados- en esta "mezcla de excitación, sorpresa, curiosidad, incertidumbre, estado de no-saber... pero sabiendo que estaban aprendiendo algo, que eso era importante" (Onsès, 2018: 248). Y no sólo durante los encuentros en la escuela. La tesis también se escribió desde este estado. La tesis -y yo con ella- creciendo como un rizoma, haciéndose/me y deshaciéndose/me según el propio devenir de la tesis:

Yo también me he permitido romperme, alterarme, conectarme, reconfigurarme, dejar entrar y salir 'cosas', dejarme llevar o no por líneas de fuga y, sobre todo, tener "la confianza en que algo saldrá de todo ello, aunque uno no esté aun totalmente seguro de qué se trata" (Rajchman, 2007, p. 12) (Onsès, 2018, p. 11).

\section{Otros modos de investigar}

Los dos trabajos presentados proponen otros modos de investigar. No por la mera voluntad de hacerlo de forma diferente, como si de un capricho se tratara, sino porque ambos, cada uno a su manera, nos encontramos con que los formatos existentes o más convencionales no nos ayudaban a pensar nuestros respectivos procesos. Al contrario, proponían métodos y prácticas vinculados a ontologías, epistemologías y metodologías que poco o nada tenían que ver con los enfoques adoptados en nuestras tesis. Aurelio Castro se sentía distante de una investigación antropológica "clásica", mientras que Judit Onsès se piensa muy alejada de la mayoría de las investigaciones educativas cualitativas.

Esto nos llevó a leer a otras autoras (Atkinson, 2011, 2012, 2017; Barad, 2003, 2007; Braidotti, 2015; Corsín, 2014; Lather y St. Pierre, 2013) y a explorar teorías y conceptos -los nuevos materialismos y empirismos, la teoría del actor-red, la teoría de los ensamblajes- que sí nos ayudaron a pensar nuestras investigaciones desde una perspectiva poscualitativa, poshumanista y rizomática. Teorías que entienden la realidad de forma menos compartimentada, o más holística y relacional. Modos de entender la investigación más allá de una planificación dicotómica donde existen, principalmente, sujetos investigadores y objetos de investigación; donde la teoría se distingue de la práctica; donde lo natural se opone a lo cultural, etc. 
Además, estos nuevos modos propuestos no sólo tienen en cuenta a los sujetos en la investigación, sino que también prestan atención a los discursos que circulan, a las relaciones que se dan, a lo material, a lo pensable o impensable, a lo conocido o desconocido, etc. Todo ello, al igual que los sujetos, no cabe comprenderlo como entidades fijas y delimitadas, sino como entes activos de la investigación. Seres en movimiento, que se ven afectados y afectan a los acontecimientos que se van sucediendo y solapando a lo largo del trabajo de campo.

De ahí el concepto clave que articula ambas investigaciones: el devenir. La investigación poscualitativa pretende establecer y encontrar nuevas formas de dar cuenta de las ausencias presentes en el continuo devenir natural-cultural del mundo (Higgins, 2017), entendiendo la realidad desde su complejidad y sus multiplicidades. La investigación como un devenir y los sujetos como un devenir (Deleuze y Guattari, 2004; Braidotti, 1994). No se es investigador/a: se devieneinvestigador/a. Y la investigación y la tesis van deviniendo. Porque por mucho que uno/a prepare unos modos de hacer y de continuarla, los acontecimientos y diferentes sucesos suscitan que ésta tome otras direcciones. Entender la investigación como un enmarañamiento de procesos, relaciones y movimientos, hace que también nosotros como investigadores nos tengamos que ir recolocando, desplazando y moviendo sobre el terreno.

Así pues, la investigadora deviene en la investigación, que a su vez está deviniendo. Se entiende el todo como un constante afectarse y, por lo tanto, moverse, responder a esas afectaciones múltiples y multidireccionales. Y no sólo deviene el sujeto investigador, sino también:

- Los espacios: las salas de proyección en un caso y los espacios de la escuela en el otro.

- Las personas implicadas: los asistentes a las diferentes sesiones del cinefórum cambian, van afectando el curso de cada proyección y son afectados por la proyección y lo que acontece allí; por su lado, los estudiantes de primaria también afectan a los procesos de documentación visual y son afectados por el encuentro entre ellos, las cámaras, la investigadora, etc.

- Lo material: las butacas o mobiliario escolar, el grado de iluminación de la sala, la densidad del aire, los cuerpos de los asistentes/ participantes, sus pensamientos, lo que dicen, sienten, hacen. 
Finalmente, esta posición conlleva pensar la ontología, la epistemología, la metodología y la ética como un enmarañamiento. Porque una afecta a la otra, y la otra a la otra y a la de más allá. Todas estas dimensiones intraactúan entre ellas y se (re)configuran. No se pueden pensar por separado. Judit habla de rizoma y Aurelio de estado de torsión; no obstante, ambos se basan en un devenir que no solo afecta el trabajo de campo, sino también la propia escritura final de sus tesis. Cada una de ellas pedían cierta experimentación, el buscar estructuras y modos de escritura diferentes a 'capítulos' convencionales. En suma, buscar otras estrategias de dar cuenta, aunque fuese desde un formato escrito; experimentar con las citas, con las conexiones entre partes, con los diálogos con las fuentes consultadas, y poner en relación experiencias y conocimientos situados con descubrimientos, ideas y pensamientos nuevos.

Todo ello implica asumir riesgos, aceptar miedos y afrontar la inseguridad derivada de no saber (Rogoff, 2006), incorporando todo ello a la investigación y a la tesis; confiar en la posición onto-epistemológica adoptada; responsabilizarse de cómo respondemos a la investigación y a la tesis en cada momento, en cada encuentro y en sus devenires; y ser conscientes de que con nuestras aportaciones trataremos de agrandar un poco la fisura que se va abriendo camino poco a poco en el campo de las ciencias sociales: una vía propositiva hacia otros modos de investigar.

\section{Referencias bibliográficas}

Atkinson, D. (2011). Art, Equality and Learning. Pedagogies Against the State. Rotterdam /Boston / Taipei: Sense Publishers.

Atkinson, D.(2012). Contemporary Art and Art in Education: The New, Emancipation and Truth. The International Journal of Art \& Design Education (iJADE), 31 (1), 5-18. Recuperado 1 mayo 2017 desde: http://onlinelibrary.wiley.com/doi/10.1111/j.14768070.2012.01724.x/epdf

Atkinson, D. (2017). Without Criteria: Art and Learning and the Adventure of Pedagogy. The International Journal of Art \& Design Education (iJADE), 36 (2), 141-152. Recuperado 1 Mayo 2017 desde http://onlinelibrary.wiley.com.sire.ub.edu/doi/10.1111/ jade.12089/epdf

Atkinson, D. (2018). Art, Pedagogies and Becoming: The Force of Art and the Individuation of New Worlds. En Linda Knight and Alexandra Lasczik Cutcher (eds.), ArtsResearch-Education: Connections and Directions (3-16), Springer. DOI 10.1007/9783-319-61560-8.

Barad, K. (1996). Meeting the Universe Halfway. Realism and Social Constructivism 
La investigación sobre educación como desplazamiento: no saber, abrirse, devenir

Without Contradiction. En Lynn Hankinson Nelson y Jack Nelson (eds.), Feminism, Science, and the Philosophy of Science (pp. 161-194). Dordrecht / Boston / Londres: Kluwer Academic Publishers.

Barad, K. (2003). Posthumanist Performativity: Toward an Understanding of How Matter Comes to Matter. Journal of Women in Culture and Society, 28 (3), 801-831.

Barad, K. (2007). Meeting the Universe Halfway. Durham y Londres: Duke University Press.

Braidotti, R. (1994). Nomadic subjects. Embodiment and sexual difference in contemporary feminist theory. New York: Columbia University Press.

Braidotti, R. (2015). Lo Posthumano (1a ed.; Juan C. Gentile-Vitale, trad.). Barcelona: Gedisa. (Trabajo original publicado en 2013).

Capra, F. (2003). Las conexiones ocultas: implicaciones sociales, medioambientales, económicas y biológicas de una nueva visión del mundo (David Sempau, trad.). Barcelona: Anagrama. (Trabajo original publicado en 2000).

Corsín, A. (2014). The Right to Infrastructure: A Prototype for Open Source Urbanism. Environment and Planning D: Society and Space 32 (2): 342-362.

Corsín, A., y Estalella, A. (2013). Asambleas al aire: la arquitectura ambulatoria de una política en suspensión. Revista de Antropología Experimental 13, 73-88.

Corsín, A., y Estalella, A. (2016). Ecologies in Beta: The City as Infrastructure of Apprenticeships. En P. Harvey, C. Bruun Jensen y A. Morita (ed.) Infrastructure and Social Complexity: A Routledge Companion, (pp.141-156). Londres: Routledge.

Deleuze, G., y Guattari, F. (2004). Mil mesetas. Capitalismo y esquizofrenia (6a ed., José Vázquez Pérez con la colaboración de Umbelina Larraceleta, trad.). Valencia: PreTextos. (Trabajo original publicado en 1980).

Domingo-Coscollola, M.; Onsès-Segarra, J. y Sancho-Gil, J.M. (2018). La cultura DIY en educación primaria. Aprendizaje transdisciplinar, colaborativo y compartido en Hub DIYLab. Revista de Investigación Educativa, 36(2), 491-508. DOI: https://doi. org/10.6018/rie.36.2.304421

Ellsworth, E. (2005). Places of Learning: Media, Architecture, Pedagogy. New York, NY: Routledge.

Estalella, A., y Sánchez Criado, T. (2016). Experimentación etnográfica: infraestructuras de campo y re-aprendizajes de la antropología. Revista de Dialectología y Tradiciones Populares LXXI (1), 9-30.

Foucault, M. (1997). Ethics, Subjectivity and Truth. New York: The New Press.

Greene, J. C. (2013). On rhizomes, lines of flight, mangles, and other assemblages, International Journal of Qualitative Studies in Education, 26 (6), 749-758, DOI:10.1080/0 9518398.2013.788763

Higgins, Marc (2017). Post-qualitative mo(ve)ments: Concluding remarks on methodological response-abilities and being wounded by thought. Reconceptualizing Educational Research Methodology, 8(3), 89-101

Holmes, D. R., y Marcus, G. E. (2008). Collaboration Today and the Re-imagination of the Classic Scene of Fieldwork Encounter. Collaborative Anthropologies, 1 (1), 81-101.

Hoyuelos, A. (2007). Aula de Infantil. [Versión electrónica]. Revista Aula de Infantil, 39. 
La investigación sobre educación como desplazamiento: no saber, abrirse, devenir

Recuperado 8 julio 2018 desde http://caps.educacion.navarra.es/infantil/attachments/article/112/documentacion-como-narracion-y-argumentacion.pdf

Kanuha, V. K. (2000). 'Being' Native versus 'Going Native': Conducting Social Work Research as an Insider. Social Work 45 (5), 439-447.

Krechevski, M., Mardell, B., Rivard, M., y Wilson, D. G. (2013). Visible Learners. Promoting Reggio-Inspired Approaches in all Schools. San Francisco: Jossey-Bass.

Lafuente, A., Alonso, A., y Rodríguez, J. (2013). ¡Todos sabios! Ciencia ciudadana y conocimiento expandido. Madrid: Cátedra.

Lather, P. (1990). Postmodernism and the Human Sciences. The Humanistic Psychologist, 18, 64-84.

Lather, P., y St. Pierre, E. (2013). Post-qualitative research. International Journal of Qualitative Studies in Education, 26(6), 629-633, DOI 10.1080/09518398.2013.788752

Lia, A., Iozzelli, S., Davoli, M., y Tognetti, G. (2013). Documentar: afinar els ulls per captar moments (4a ed., Àngels Polo, trad.). Barcelona: Associació de mestres de Rosa Sensat.

Malinowski, B. (1922). Argonauts of the Pacific. New York: Holt, Rinehart \& Winston.

Marcus, G. E. (2013). Experimental Forms for the Expression of Norms in the Ethnography of the Contemporary. Hau: Journal of Ethnographic Theory, 3 (2), 197-217.

Martin, A. D., y Kamberelis, G. (2013). Mapping not tracing: Qualitative educational research with political teeth. International Journal of Qualitative Studies in Education, 26 (6), 668-679.

Onsès, J. (2018). Documentación visual en los fenómenos de aprendizaje con estudiantes de primaria. Una indagación rizomática difractiva desde las teorías 'post' Barcelona: Universitat de Barcelona. Tesis doctoral no publicada.

Rajchman, J. (2007). Deleuze. Un mapa (1a reimpresión de la 1a ed. en español; Elena Marengo, trad.). Buenos Aires: Nueva Visión. (Trabajo original publicado en 2000).

Rogoff, I. (2006). Academy as potentiality. En F. Allen (Ed.), Education, Documents of Contemporary Art, 4-9. London: Whitechapel Gallery.

Sánchez de Serdio, A. (2010). Políticas de lo concreto: producción cultural colaborativa y modos de organización. En A. Collados, G. Kester, J. Rodrigo, Y. Romero y A. Sánchez de Serdio, Transductores 1. Pedagogías colectivas y políticas espaciales (pp. 44-65). Granada: Centro José Guerrero.

Springgay, S., Irwin, R. L., y Wilson, S. (2005). A/r/tography as Living Inquiry Through Art and Text. Qualitative Inquiry, 11(6), 897-912.

St. Pierre, E. A. (2011). Post Qualitative Research: The Critique and the Coming After. En N. K. Denzin e Y. S. Lincoln (eds.) The Sage Handbook of Qualitative Research (4a ed. Revisada, (pp. 611-625). Thousand Oaks: SAGE Publications Inc.

St. Pierre, E. A. (2013). The posts continue: becoming. International Journal of Qualitative Studies in Education, 26(6), 646-657, DOI 10.1080/09518398.2013.788754

St. Pierre, E. A. (2014). A Brief and Personal History of Post Qualitative Research Toward "Post Inquiry". Journal of Curriculum Theorizing, 30(2), 2-20. Recuperado 7 abril 2018 desde http://journal.jctonline.org/index.php/jct/article/view/521/stpierre.pdf

St. Pierre, El. A. (2016). The Empirical and the New Empiricism. Cultural Studies Critical Methodologies 16(2), 111-124. 
St. Pierre, E. A., Jackson, A.Y., y Mazzei, L. A. (2016). New Empiricisms and New Materialisms: Conditions for New Inquiry. Cultural Studies Critical Methodologies 16(2), 99-110.

Tartás, C., y Guridi, R. (2013). Cartografías de la memoria. Aby Warburg y el Atlas Mnemosyne. EGA. Revista de expresión gráfica arquitectónica, 21, 226-235.

Taylor, C. A. (2016). Close Encounters of a Critical Kind: A Diffractive Musing In/Between New Material Feminism and Object-Oriented Ontology. Cultural Studies, 16(2), 201212.

Xarxa Territorial d'Educació Infantil a Catalunya (2009a). Documentar, una mirada nova. Barcelona: Associació de mestres de Rosa Sensat.

Xarxa Territorial d'Educació Infantil a Catalunya (2009b). Documentar la vida dels infants a l'escola (3a ed.). Barcelona: Associació de mestres de Rosa Sensat.

\section{Agradecimientos}

Cinefórum de la Asamblea de Vecinas de Poble Sec

Esbrina - Subjetividades, visualidades y entornos educativos contemporáneos (2017SGR 1248).

REUNI+D -Red Universitaria de Investigación e Innovación Educativa. Cambios Sociales y Retos para la Educación en la Era Digital (EDU201568718-REDT). 
